\title{
Expression and significance of serum soluble fms-like tyrosine kinase 1 (sFlt-1), CXC chemokine ligand 16 (CXCL16), and lipocalin 2 (LCN-2) in pregnant women with preeclampsia
}

\author{
Lingling $\mathrm{Li}^{1,2 \#}$, Bo Ling ${ }^{1,2 \#}$, Jie Mei ${ }^{1,2}$, Yuju Wang ${ }^{1,2}$, Jian Zhang ${ }^{1,2}$, Xiaolan Zhao ${ }^{1,2}$ \\ ${ }^{1}$ Department of Gynaecology and Obstetrics, Sichuan Provincial People's Hospital, University of Electronic Science and Technology of China, \\ Chengdu, China; ${ }^{2}$ Chinese Academy of Sciences Sichuan Translational Medicine Research Hospital, Chengdu, China \\ Contributions: (I) Conception and design: J Zhang, X Zhao; (II) Administrative support: J Zhang, X Zhao; (III) Provision of study materials or \\ patients: L Li, B Ling; (IV) Collection and assembly of data: L Li, B Ling; (V) Data analysis and interpretation: J Mei, Y Wang; (VI) Manuscript \\ writing: All authors; (VII) Final approval of manuscript: All authors. \\ \#These authors contributed equally to this work. \\ Correspondence to: Jian Zhang; Xiaolan Zhao. Department of Gynaecology and Obstetrics, 32\#, West Road Section 2, First Ring Road, Chengdu, \\ China. Email: ellajeff@126.com; zhao_xiaolan@126.com.
}

Background: To explore the value of serum soluble fms-like tyrosine kinase 1 (sFlt-1), CXC chemokine
ligand 16 (CXCL16), and lipocalin 2 (LCN-2) in the diagnosis and grading of preeclampsia (PE).

Methods: A total of 186 patients with PE diagnosed and treated in our hospital were included. According to the disease severity, the patients were divided into the mild PE group (99 cases) and the severe PE group (87 cases). A total of 72 healthy pregnant women who underwent antenatal care were selected as the healthy control group. The levels of serum sFlt-1, CXCL16, and LCN-2 before medication were compared among the patients, and the diagnosis and grading value of the above 3 indicators were analyzed.

Results: For PE patients vs. healthy controls, the levels of sFlt-1 (132.71 \pm 14.49 vs. $68.43 \pm 9.28 \mu \mathrm{g} / \mathrm{L})$, CXCL16 (2.15 \pm 0.35 vs. $0.61 \pm 0.12 \mu \mathrm{g} / \mathrm{L})$, and $\mathrm{LCN}-2(70.81 \pm 8.25$ vs. $19.22 \pm 3.14 \mu \mathrm{g} / \mathrm{L})$ were all significantly higher in $\mathrm{PE}$ patients than in the healthy controls $(\mathrm{P}<0.05)$. For severe $\mathrm{PE}$ vs. mild $\mathrm{PE}$, the levels of sFlt-1 $(142.16 \pm 20.23$ vs. $124.41 \pm 10.36 \mu \mathrm{g} / \mathrm{L})$, CXCL16 (2.87 \pm 0.59 vs. $1.51 \pm 0.28 \mu \mathrm{g} / \mathrm{L})$, and LCN-2 $(90.76 \pm 10.16$ vs. 53.27 $\pm 6.19 \mu \mathrm{g} / \mathrm{L})$ in the severe $\mathrm{PE}$ group were higher than those in the mild $\mathrm{PE}$ group $(\mathrm{P}<0.05)$. Receiver operating characteristic curve (ROC) analysis showed that when the cut-off values of sFlt-1, CXCL16, and LCN-2 were 99.65, 1.36, and $0.84 \mu \mathrm{g} / \mathrm{L}$, respectively, the diagnostic efficacy of PE was the highest. With these cut-off values, the diagnostic sensitivities of sFlt-1, CXCL16, and LCN-2 were 86.67\%, 73.33\%, and 93.33\%, respectively. The specificities of sFlt-1, CXCL16, and LCN-2 were $80.00 \%, 86.67 \%$, and $60.00 \%$, respectively. The areas under the curves (AUC) of sFlt-1, CXCL16, and LCN-2 were 0.764, 0.769, and 0.831, respectively. When the cut-off values for sFlt-1, CXCL16, and LCN-2 were 135.16, 2.24, and 70.38 $\mu \mathrm{g} / \mathrm{L}$, respectively, the efficacy was the highest in distinguishing mild and severe PE. With these cut-off values, the AUC values of sFlt-1, CXCL16, and LCN-2 were 0.837, 0.808, and 0.869, respectively.

Conclusions: sFlt-1, CXCL16, and LCN-2 have certain significance in the diagnosis and grading of PE. Among them, LCN-2 has the highest correlation with the diagnosis and grading of PE.

Keywords: Soluble fms-like tyrosine kinase 1 (sFlt-1); CXC chemokine ligand 16 (CXCL16); lipocalin 2 (LCN-2); preeclampsia $(\mathrm{PE})$

Submitted May 12, 2021. Accepted for publication Jun 24, 2021.

doi: 10.21037/apm-21-1553

View this article at: https://dx.doi.org/10.21037/apm-21-1553

(c) Annals of Palliative Medicine. All rights reserved. 


\section{Introduction}

Preeclampsia $(\mathrm{PE})$ is a multisystem disease that occurs during pregnancy, and often manifests as transient hypertension, systemic endothelial dysfunction, and proteinuria after 20 weeks of pregnancy (1). The incidence of the disease is between $5 \%$ and $8 \%$ (2). PE patients are usually severely ill and progress rapidly. Therefore, $\mathrm{PE}$ has become one of the main causes of death among pregnant women worldwide (3). Currently, it is believed that PE is caused by insufficient placental perfusion-related ischemia and hypoxia, and subsequent secretion of toxic factors into blood vessels (4). Clinically, 24-hour proteinuria and clinical symptoms are used to diagnose and grade the severity of PE. However, the collection of 24-hour proteinuria specimens makes it difficult to diagnose and grade the disease in time. Achieving a fast diagnosis of $\mathrm{PE}$ has become a clinical research hotspot (5). In this study, serum soluble fms-like tyrosine kinase 1 (sFlt-1), CXC chemokine ligand 16 (CXCL16), and lipocalin 2 (LCN-2) levels were assessed with enzyme-linked immunosorbent assay (ELISA). The accuracy and efficiency of the 3 indicators in diagnosing and grading PE were discussed. We present the following article in accordance with the STARD reporting checklist (available at https://dx.doi.org/10.21037/apm-21-1553).

\section{Methods}

\section{Participants}

A total of $186 \mathrm{PE}$ patients who received treatment in our hospital from January 2019 to December 2020 and 72 healthy parturients who underwent antenatal care during the same period were enrolled. The inclusion criteria for PE patients were as follows: diagnosed with $\mathrm{PE}$ (6); singleton pregnancy; natural conception. The exclusion criteria were as follows: patients with coagulation dysfunction; had a history of hypertension or heart disease; under blood pressure controlrelated treatments; incomplete clinical data.

All procedures performed in this study involving human participants were in accordance with the Declaration of Helsinki (as revised in 2013). The study was approved by the ethics committee of Sichuan Provincial People's Hospital. Written informed consent was signed by all participants.

\section{PE degree classification standard}

The 186 cases of PE patients were all in the PE group, and the PE patients were divided into the mild and severe $\mathrm{PE}$ group according to the disease severity (7). Mild PE was defined as: blood pressure $\geq 140 / 90 \mathrm{mmHg}$ measured 4 hours after 20 weeks of pregnancy; random proteinuria (+) or urine protein $\geq 300 \mathrm{mg} / 24 \mathrm{~h}$. Other potential symptoms in mild PE patients included headache and abdominal discomfort. Severe PE was defined as: blood pressure measurement $\geq 160 / 110 \mathrm{mmHg}$; random proteinuria $\geq(++)$ or urine protein $\geq 2.0 \mathrm{~g} / 24 \mathrm{~h}$. Other symptoms of severe PE patients included persistent upper abdominal discomfort or persistent headache and visual disturbance.

\section{Sample collection and tests}

Five $\mathrm{mL}$ fasting blood from the anterior elbow vein of $\mathrm{PE}$ patients before treatment was collected, and $5 \mathrm{~mL}$ fasting blood from the anterior elbow vein of healthy pregnant women on the day of physical examination was collected. After centrifugation at $3,200 \mathrm{r} / \mathrm{min}$, the serum was separated and placed in a freezer at $-80{ }^{\circ} \mathrm{C}$ until use. The ELISA method was used to determine sFlt-1, CXCL16, and LCN-2 levels. The kit was taken out from the refrigerator 15 minutes before the test and placed at room temperature. Sample diluent $(50 \mu \mathrm{L})$ was added to each $10 \mu \mathrm{L}$ serum sample. After mixing well, the samples were incubated at $37^{\circ} \mathrm{C}$ for $15 \mathrm{~min}$. After incubation, the liquid was discarded, and the wells were dried with absorbent papers. The wells were then washed 3 times by adding the washing solution for $30 \mathrm{~s}$, pouring off the liquid, then drying with absorbent papers. Then, $50 \mu \mathrm{L}$ of enzymelabeled working solution was added to each well and incubated at $37^{\circ} \mathrm{C}$ for 15 minutes. After the incubation, the plate washing was repeated 3 times as mentioned. After drying, $50 \mu \mathrm{L}$ of chromogenic agent $\mathrm{A}$ and $\mathrm{B}$ were added sequentially. After mixing, the wells were incubated in a dark environment at $37^{\circ} \mathrm{C}$ for $20 \mathrm{~min}$. Finally, $50 \mu \mathrm{L}$ of stop solution was added, and the absorbance value (OD value) of each well was measured within 10 minutes after termination. The OD values of the blank control and standards were tested to generate a standard curve. The final sample concentrations were calculated as tested values $\times$ the dilution ratio.

\section{Statistical analysis}

The data obtained were analyzed by SPSS 22.0 software. Measurement data were expressed as $\bar{x} \pm \mathrm{s}$, the comparison between groups was performed by the $t$ test, and the general 
Table 1 General data of patients

\begin{tabular}{lcccccc}
\hline Group & $\mathrm{n}$ & Age (year) & $\mathrm{BMI}\left(\mathrm{kg} / \mathrm{m}^{2}\right)$ & Gestation time $($ week) & Systolic pressure $(\mathrm{mmHg})$ & Diastolic pressure $(\mathrm{mmHg})$ \\
\hline Healthy & 72 & $30.11 \pm 2.16$ & $24.89 \pm 2.54^{\#}$ & $32.41 \pm 2.06$ & $110.19 \pm 8.12^{\#}$ & $75.13 \pm 5.91^{\#}$ \\
Mild PE & 99 & $29.98 \pm 2.55$ & $27.79 \pm 3.05^{\star}$ & $33.15 \pm 2.58$ & $141.61 \pm 10.39^{\star}$ & $95.12 \pm 3.64^{\star}$ \\
Severe PE & 87 & $30.47 \pm 2.07$ & $27.88 \pm 2.94^{\star}$ & $32.68 \pm 2.64$ & $157.46 \pm 15.14^{\star \#}$ & $103.15 \pm 2.16^{\star \#}$ \\
$F$ & & 1.109 & 27.133 & 1.993 & 327.784 & 988.984 \\
P & & 0.331 & $<0.01$ & 0.138 & $<0.01$ & $<0.01$ \\
\hline
\end{tabular}

Compared to healthy controls, ${ }^{*} \mathrm{P}<0.05$; compared to the mild $\mathrm{PE}$ group, ${ }^{*} \mathrm{P}<0.05$. BMI, body mass index; $\mathrm{PE}$, preeclampsia.

Table 2 Comparison of the levels of sFlt-1, CXCL16, and LCN-2 between the PE group and the healthy group $(\bar{x} \pm \mathrm{S})$

\begin{tabular}{lcccc}
\hline Group & $\mathrm{n}$ & $\mathrm{sFlt}-1(\mu \mathrm{g} / \mathrm{L})$ & $\mathrm{CXCL} 16(\mu \mathrm{g} / \mathrm{L})$ & $\mathrm{LCN}-2(\mu \mathrm{g} / \mathrm{L})$ \\
\hline Healthy & 72 & $68.43 \pm 9.28$ & $0.61 \pm 0.12$ & $19.22 \pm 3.14$ \\
$\mathrm{PE}$ & 186 & $132.71 \pm 14.49$ & $2.15 \pm 0.35$ & $70.81 \pm 8.25$ \\
$t$ & & 34.947 & 36.477 & 51.583 \\
$\mathrm{P}$ & & $<0.01$ & $<0.01$ & $<0.01$
\end{tabular}

sFIt-1, soluble fms-like tyrosine kinase 1; CXCL16, CXC chemokine ligand 16; LCN-2, lipocalin 2; PE, preeclampsia.

data of the 3 groups of patients were analyzed by one-way analysis of variance. The sensitivity and specificity of sFlt-1, CXCL16, and LCN-2 in the diagnosis and grading of PE were analyzed with the receiver operating characteristic (ROC) curve. $\mathrm{P}<0.05$ indicated that the difference was statistically significant.

\section{Results}

\section{General information of participants}

There were 99 cases in the mild PE group and 87 cases in the severe PE group. The body mass index (BMI), systolic blood pressure, and diastolic blood pressure of the $\mathrm{PE}$ patients were higher than those of the healthy group $(\mathrm{P}<0.05)$, and the systolic and diastolic blood pressures of the severe $\mathrm{PE}$ group were higher than those of the mild group $(\mathrm{P}<0.05)$. No significant difference was observed in the age and gestational age of the 3 groups of participants $(\mathrm{P}>0.05$; Table 1).

\section{Comparison of sFlt-1, CXCL16, and LCN-2 levels between the PE group and the healthy group}

The levels of sFlt-1, CXCL16, and LCN-2 in the PE
Table 3 Comparison of the levels of sFlt-1, CXCL16, and LCN-2 between the mild PE group and the severe PE group $(\bar{x} \pm \mathrm{S})$

\begin{tabular}{lcccc}
\hline Group & $\mathrm{n}$ & $\mathrm{sFlt}-1(\mu \mathrm{g} / \mathrm{L})$ & $\mathrm{CXCL} 16(\mu \mathrm{g} / \mathrm{L})$ & $\mathrm{LCN}-2(\mu \mathrm{g} / \mathrm{L})$ \\
\hline Mild PE & 99 & $124.41 \pm 10.36$ & $1.51 \pm 0.28$ & $53.27 \pm 6.19$ \\
Severe PE & 87 & $142.16 \pm 20.23$ & $2.87 \pm 0.59$ & $90.76 \pm 10.16$ \\
$t$ & & 7.663 & 20.467 & 30.789 \\
$\mathrm{P}$ & & $<0.01$ & $<0.01$ & $<0.01$ \\
\hline
\end{tabular}

sFlt-1, soluble fms-like tyrosine kinase 1; CXCL16, CXC chemokine ligand 16; LCN-2, lipocalin 2; PE, preeclampsia.

group were higher than those in the healthy group, and the difference was statistically significant $(\mathrm{P}<0.05$; Table 2$)$.

Comparison of sFlt-1, CXCL16, and LCN-2 levels between the mild PE group and the severe PE group

The levels of sFlt-1, CXCL16, and LCN-2 in the severe $\mathrm{PE}$ group were all higher than those in the mild $\mathrm{PE}$ group, and the difference was statistically significant $(\mathrm{P}<0.05$; Table 3).

\section{The value of sFlt-1, CXCL16, and LCN-2 in the diagnosis} of $P E$

The ROC curve showed that when the sFlt- 1 cut-off value was $99.65 \mu \mathrm{g} / \mathrm{L}$, the area under the curve (AUC), sensitivity, and specificity in diagnosing PE were $0.764,86.67 \%$, and $80.00 \%$, respectively. When the CXCL16 cut-off value was $1.36 \mu \mathrm{g} / \mathrm{L}$, the AUC, sensitivity, and specificity in diagnosing PE were $0.769,73.33 \%$, and $86.67 \%$ respectively. When the LCN-2 cut-off value was $0.84 \mu \mathrm{g} / \mathrm{L}$, the AUC, sensitivity, and specificity in diagnosing PE were 0.831 , $93.33 \%$, and $60.00 \%$, respectively, as shown in Figure 1 and Table 4. 


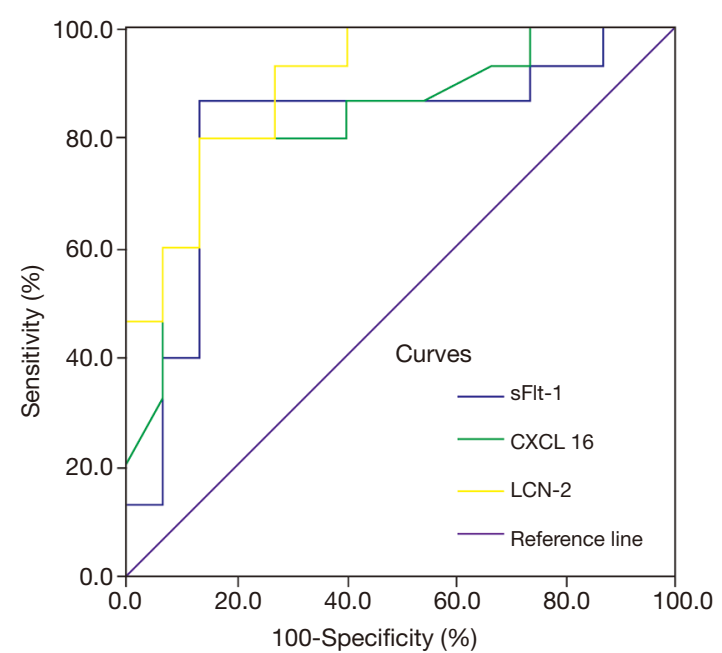

Figure 1 Receiver operating characteristic (ROC) curves of soluble fms-like tyrosine kinase 1 (sFlt-1), CXC chemokine ligand 16 (CXCL16) and lipocalin 2 (LCN-2) in the diagnosis of preeclampsia $(\mathrm{PE})$.

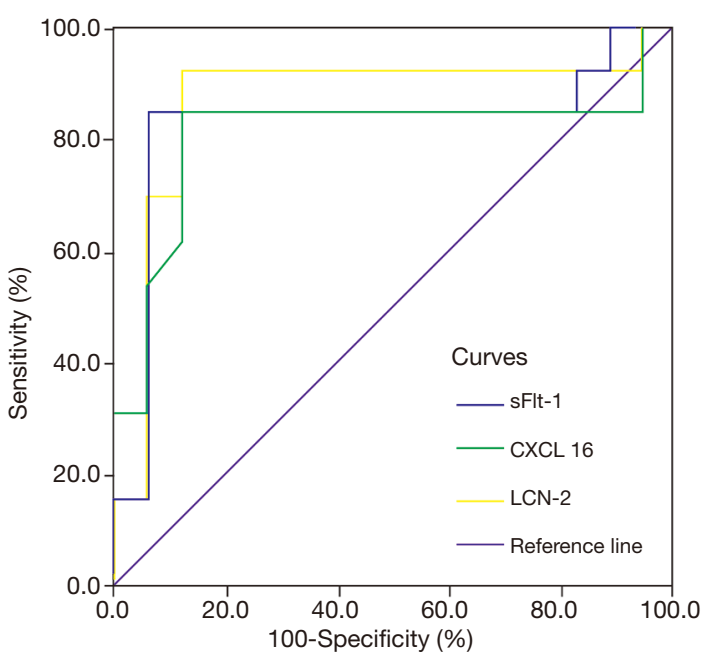

Figure 2 Receiver operating characteristic (ROC) curves of soluble fms-like tyrosine kinase 1 (sFlt-1), CXC chemokine ligand 16 (CXCL16) and lipocalin 2 (LCN-2) in the grading of mild and severe preeclampsia $(\mathrm{PE})$.

Table 4 Value of sFlt-1, CXCL16, and LCN-2 in the diagnosis of PE

\begin{tabular}{lcccccc}
\hline Indicator & Cut-off & AUC & Sensitivity (\%) & Specificity (\%) & Youden index & 95\% Cl \\
\hline sFlt-1 & $>99.65$ & 0.764 & 86.67 & 80.00 & 0.667 & $0.575-0.899$ \\
CXCL16 & $>1.36$ & 0.769 & 73.33 & 86.67 & 0.600 & $0.580-0.902$ \\
LCN-2 & $>0.84$ & 0.831 & 93.33 & 60.00 & 0.53 & $0.650-0.942$
\end{tabular}

sFlt-1, soluble fms-like tyrosine kinase 1; CXCL16, CXC chemokine ligand 16; LCN-2, lipocalin 2; PE, preeclampsia; AUC, area under curve; $\mathrm{Cl}$, confidence interval.

\section{The diagnostic value of sFlt-1, CXCL16, and LCN-2 in discriminating mild and severe $P E$}

The ROC curve showed that when the cut-off value for sFlt-1 was $135.16 \mu \mathrm{g} / \mathrm{L}$, the AUC, sensitivity, and specificity for distinguishing mild and severe $\mathrm{PE}$ were 0.837 , $84.62 \%$, and $94.12 \%$, respectively. When the cut-off value for CXCL16 was $2.24 \mu \mathrm{g} / \mathrm{L}$, the AUC, sensitivity, and specificity were $0.808,84.62 \%$, and $88.24 \%$, respectively. When the cut-off value for LCN-2 was $70.38 \mu \mathrm{g} / \mathrm{L}$, the AUC, sensitivity, and specificity were $0.869,92.31 \%$, and $88.24 \%$, respectively (Figure 2 and Table 5).

\section{Discussion}

$\mathrm{PE}$ is a multisystem dysfunction that happens in women after week 20 of gestation (4). Induced by placental hypoperfusion, PE progression leads to changes in placental microenvironment, releasing of cytokines, and finally resulting in proteinuria and maternal hypertension. There was no targeted treatment for PE, antihypertensive, spasmolysis and sedation are usually used, termination of pregnancy was also a choice to severe conditions.

PE progresses rapidly. There are currently no specific laboratory indicators to diagnose and grade the severity of PE patients, which may have an impact on the subsequent treatment of patients $(8,9)$. The anti-angiogenic factor sFlt-1 is secreted by the placenta and is involved in vascular regulation. It can cause placental angiogenesis and cause vascular endothelial damage $(9,10)$. CXCL16 is a multifunctional protein which is soluble in the cytoplasm or bound in the membrane. CXCL16 is expressed in T cells, B cells, epithelial cells, macrophages, fibroblasts, endothelial cells, and vascular smooth muscle cells (11). LCN-2 is a widely distributed member of the lipocalin family. LCN-2 can mediate inflammation, inhibit cell apoptosis, and promote tumor cell metastasis. The expression of $\mathrm{LCN}-2$ 
Table 5 Diagnostic value of sFlt-1, CXCL16, and LCN-2 in grading mild and severe PE

\begin{tabular}{lcccccr}
\hline Indicator & Cut-off & AUC & Sensitivity (\%) & Specificity (\%) & Youden index & $95 \%$ Cl \\
\hline sFlt-1 & $>135.16$ & 0.837 & 84.62 & 94.12 & 0.787 & $0.657-0.946$ \\
CXCL16 & $>2.24$ & 0.808 & 84.62 & 88.24 & 0.729 & $0.623-0.928$ \\
LCN-2 & $>70.38$ & 0.869 & 92.31 & 88.24 & 0.805 & $0.695-0.964$ \\
\hline
\end{tabular}

sFlt-1, soluble fms-like tyrosine kinase 1; CXCL16, CXC chemokine ligand 16; LCN-2, lipocalin 2; PE, preeclampsia; AUC, area under curve; $\mathrm{Cl}$, confidence interval.

is increased in a variety of inflammatory reactions such as enteritis, peritonitis, and atherosclerosis $(12,13)$.

In this study, the levels of sFlt-1, CXCL16, and LCN-2 in the $\mathrm{PE}$ group were higher than those in the healthy group. The levels of sFlt-1, CXCL16, and LCN-2 in the severe $\mathrm{PE}$ group were higher than those in the mild $\mathrm{PE}$ group. The ROC curve results in this study show that sFlt-1, CXCL16, and LCN-2 all have good sensitivity for the diagnosis of PE, among which $\mathrm{LCN}-2$ is the best for the diagnosis of $\mathrm{PE}$, as it reached a diagnostic sensitivity of $93.33 \%$ and an AUC of 0.831 . Insufficient blood supply to the placenta leads to the increase in sFlt-1 content, and the increase in sFlt-1 leads to angiogenesis dysfunction, damaged endothelial cells, and subsequent inflammation and PE (9). Inflammation and endothelial damage can induce high expression of LCN-2, which can possibly cause vascular sclerosis to aggravate PE (14). LCN-2 can combine with matrix metalloprotein-9 (MMP-9) to form a heterodimer protein (15). The reduced decomposition or activation of MMP-9 during endothelial dysfunction promotes vascular smooth musclerelated vascular remodeling (16), which can aggravate endothelial dysfunction and promote PE. LCN-2 is also increased in insulin-resistant individuals (12), who are often more susceptible to PE (17). Furthermore, LCN-2 has been shown to be an indicator and key player in renal injury (18), and the multiple organ dysfunction syndrome caused by PE may promote renal injury-related LCN-2 expression. All these factors may possibly explain why LCN-2 had a higher AUC and sensitivity for the diagnosis of $\mathrm{PE}$ among the 3 indicators. As a chemokine, CXCL16 can participate in inflammatory responses to aggravate vascular endothelial damage and the subsequent inflammatory response (19). CXCL16 has a high affinity with its receptors. The activation of CXCL16 enables recognition of lymphocytes to vascular endothelial cells (20), thus potentially aggravating vascular endothelial damage. We also showed that sFlt-1, CXCL16, and LCN-2 are satisfactory indicators for diagnosing and grading PE, indicating that they are all potential key players in the pathogenesis of $\mathrm{PE}$.

In summary, the levels of sFlt-1, CXCL16, and LCN-2 are significantly higher in $\mathrm{PE}$ patients. The 3 indicators can also discriminate between severe PE patients and mild PE patients, among which LCN-2 has the highest correlation with the diagnosis and grade of $\mathrm{PE}$.

\section{Acknowledgments}

Funding: None.

\section{Footnote}

Reporting Checklist: The authors have completed the STARD reporting checklist. Available at https://dx.doi. org/10.21037/apm-21-1553

Data Sharing Statement: Available at https://dx.doi. org/10.21037/apm-21-1553

Conflicts of Interest: All authors have completed the ICMJE uniform disclosure form (available at https://dx.doi. org/10.21037/apm-21-1553). The authors have no conflicts of interest to declare.

Ethical Statement: The authors are accountable for all aspects of the work in ensuring that questions related to the accuracy or integrity of any part of the work are appropriately investigated and resolved. All procedures performed in this study involving human participants were in accordance with the Declaration of Helsinki (as revised in 2013). The study was approved by the ethics committee of Sichuan Provincial People's Hospital. Written informed consent was signed by all participants.

Open Access Statement: This is an Open Access article 
distributed in accordance with the Creative Commons Attribution-NonCommercial-NoDerivs 4.0 International License (CC BY-NC-ND 4.0), which permits the noncommercial replication and distribution of the article with the strict proviso that no changes or edits are made and the original work is properly cited (including links to both the formal publication through the relevant DOI and the license). See: https://creativecommons.org/licenses/by-nc-nd/4.0/.

\section{References}

1. Girchenko P, Lahti M, Tuovinen S, et al. Cohort Profile: Prediction and prevention of preeclampsia and intrauterine growth restriction (PREDO) study. Int J Epidemiol 2017;46:1380-1g.

2. Macedo TCC, Montagna E, Trevisan CM, et al. Prevalence of preeclampsia and eclampsia in adolescent pregnancy: A systematic review and meta-analysis of 291,247 adolescents worldwide since 1969. Eur J Obstet Gynecol Reprod Biol 2020;248:177-86.

3. Hinton L, Hodgkinson J, Tucker KL, et al. Exploring the potential for introducing home monitoring of blood pressure during pregnancy into maternity care: current views and experiences of staff-a qualitative study. BMJ Open 2020;10:e037874.

4. Le Y, Ye J, Lin J. Expectant management of early-onset severe preeclampsia: a principal component analysis. Ann Transl Med 2019;7:519.

5. Shen M, Smith GN, Rodger M, et al. Comparison of risk factors and outcomes of gestational hypertension and preeclampsia. PLoS One 2017;12:e0175914.

6. Alan B, Tunç S, Agacayak E, et al. Diagnosis of preeclampsia and assessment of severity through examination of the placenta with acoustic radiation force impulse elastography. Int J Gynaecol Obstet 2016;135:43-6.

7. Cakmak M, Yilmaz H, Bağlar E, et al. Serum levels of endocan correlate with the presence and severity of preeclampsia. Clin Exp Hypertens 2016;38:137-42.

8. Duan Z, Li C, Leung WT, et al. Alterations of Several Serum Parameters Are Associated with Preeclampsia and May Be Potential Markers for the Assessment of PE Severity. Dis Markers 2020;2020:7815214.

9. Nikuei P, Rajaei M, Roozbeh N, et al. Diagnostic accuracy of sFlt1/PlGF ratio as a marker for preeclampsia. BMC Pregnancy Childbirth 2020;20:80.

10. Caillon H, Tardif C, Dumontet E, et al. Evaluation of sFlt-1/PlGF Ratio for Predicting and Improving Clinical Management of Pre-eclampsia: Experience in a Specialized
Perinatal Care Center. Ann Lab Med 2018;38:95-101.

11. Li CH, Xu LL, Zhao JX, et al. CXCL16 upregulates RANKL expression in rheumatoid arthritis synovial fibroblasts through the JAK2/STAT3 and p38/MAPK signaling pathway. Inflamm Res 2016;65:193-202.

12. Wang W, Ye S, Qian L, et al. Elevated serum lipocalin 2 levels are associated with indexes of both glucose and bone metabolism in type 2 diabetes mellitus. Endokrynol Pol 2018;69:276-82.

13. Mori K, Suzuki T, Minamishima S, et al. Neutrophil gelatinase-associated lipocalin regulates gut microbiota of mice. J Gastroenterol Hepatol 2016;31:145-54.

14. Millar SA, Anderson SI, O'Sullivan SE. Osteokines and the vasculature: a review of the in vitro effects of osteocalcin, fibroblast growth factor-23 and lipocalin-2. PeerJ 2019;7:e7139.

15. Lin CW, Tseng SW, Yang SF, et al. Role of lipocalin 2 and its complex with matrix metalloproteinase-9 in oral cancer. Oral Dis 2012;18:734-40.

16. Han Y, Sun HJ, Tong Y, et al. Curcumin attenuates migration of vascular smooth muscle cells via inhibiting NFkB-mediated NLRP3 expression in spontaneously hypertensive rats. J Nutr Biochem 2019;72:108212.

17. Kelly CB, Hookham MB, Yu JY, et al. Subclinical First Trimester Renal Abnormalities Are Associated With Preeclampsia in Normoalbuminuric Women With Type 1 Diabetes. Diabetes Care 2018;41:120-7.

18. Lee SA, Noel S, Kurzhagen JT, et al. CD4(+) T CellDerived NGAL Modifies the Outcome of Ischemic Acute Kidney Injury. J Immunol 2020;204:586-95.

19. Roh YS, Seki E. Chemokines and Chemokine Receptors in the Development of NAFLD. Adv Exp Med Biol 2018;1061:45-53.

20. Ma C, Han M, Heinrich B, et al. Gut microbiomemediated bile acid metabolism regulates liver cancer via NKT cells. Science 2018;360:eaan5931.

(English Language Editor: C. Betlazar-Maseh)

Cite this article as: Li L, Ling B, Mei J, Wang Y, Zhang J, Zhao X. Expression and significance of serum soluble fms-like tyrosine kinase 1 (sFlt-1), CXC chemokine ligand 16 (CXCL16), and lipocalin 2 (LCN-2) in pregnant women with preeclampsia. Ann Palliat Med 2021;10(7):7866-7871. doi: 10.21037/apm-211553 